\title{
The future-Everybody and everything connected
}

\author{
Gerard van OORTMERSSEN \\ Netherlands Organization for Applied Scientific Research (TNO)
}

We live in interesting times. Information and Communication Technology (ICT) is changing our life and society at an incredible pace.

It is only half a century ago that the first computers were built. At first, these were used mainly for military purposes, for science, and later on for automation of business processes. Use of computers was limited to a relatively small group of specialists. After the personal computer was introduced around 1980, computing became part of the everyday life of a larger group of people. At about the same time, the internet started to grow. Again, at first mostly among academics and the military, but after Tim Berners Lee introduced the World Wide Web, internet use for communication, searching of information, shopping, money transfer and doing business became popular at an immense scale.

At about the same time, a revolution took place in the telecom world. Or more precisely: several revolutions occurred at the same time. The telecom business was transformed into an open market, mobile phones conquered the world, and digital signal processing was introduced.

At present we witness the convergence of the telecom world and the world of internet and computers. The internet and the fixed and mobile telecom networks merge into one global digital network based on IP, the internet protocol. This network is encompassing voice, video and data communication, giving us access to computing resources, and to information, irrespective of where we are or where the resources that we want to access are located.

As a consequence, also the world of mass media, newspapers, magazines, TV and radio, is thoroughly transformed and becomes part of the internet. In the future we can, for instance, watch a TV program at any time we want, at any place. But it will also be feasible to produce our own content and broadcast our own TV

Received March 15, 2006

DOI: $10.2201 / \mathrm{NiiPi} .2006 .3 .1$ program.

What is the next step in this amazing development?

Predicting the future is a precarious activity. Nevertheless, by extrapolating the present development, new perspectives are revealed.

Chips for processing and for wireless communication continue to become smaller and cheaper. This will result in a further proliferation of intelligence in devices in our personal environment. In addition, all kind of sensors become cheap and superfluous. These sensors can be connected in sensor networks thus enabling the continuous monitoring of all kind of parameters. Application possibilities of such sensor networks are abundant: in healthcare, logistics, public security, transportation systems, agriculture, etcetera. RFID (Radio Frequency Identification) chips will be attached to many articles, so that we can follow their whereabouts.

Not only can we communicate with anybody, at any place, any time, but also will it be possible for us to communicate with devices and for machines to communicate with other machines. This new development is now often referred to as "the internet of things", but I think it is more appropriate to speak of "the internet of people and things": everybody and everything connected anytime!

Extrapolation works only to some extent. Every now and then the development line is interrupted and jumps to another level, for instance when a new, disruptive technology is introduced. A fundamental change takes place in such discontinuities, which can not be foreseen. I believe that in case of ICT development we are approaching such a fundamental change, in fact a next step in the evolution.

If we consider the evolution of the universe and of living beings, what we see is a continuously increasing complexity. In the early stages of the universe, there existed only elementary particles and simple atoms. Gradually, more complex molecules were formed. At a cer- 
tain stage, simple forms of life emerged. The transition from dead material to living beings is a very fundamental one, which required a certain degree of complexity. Life evolved, from simple, one-cell organisms, to more and more complex beings with senses to perceive the environment and a nerve system to process information of the sensory system, and to control body functions.

Evolution based on survival of the fittest stimulated progressive development. Again, a fundamental transition took place when the complexity of the nerve system reached a certain critical value: intelligence and consciousness appeared in mammals, reaching its apex, at least for the time being, in the human species.

Mankind has started to help the natural evolution through technology. At first, we have enhanced our body functions by means of all kind of tools, cars, planes etc. Now, by using ICT, we are enhancing our brains and sensory system.

The development of ICT is reminiscent to the process of evolution. We can observe a growing complexity. The short and frequent product cycles combined with the market mechanism stimulate progress through natural selection, "survival of the fittest". And the internet can be considered as an artificial nerve system. Sensor networks are analogous to the sensory perception in animals and humans.

I believe that we are on the eve of another fundamental transition, a next step in the evolution, this time realised by technology. What this step will look like I don't know, neither do I know when it will occur. I cannot predict the future. But I can imagine some possible directions.

We can be continuously online, and access vast amounts of information. But we can also communicate with anybody in the world, through voice, video conferencing, email etcetera. Not only with our relatives, friends and colleagues, but with anyone. Through web $\operatorname{logs}$, chat rooms, wiki's, and web casts we can reach everybody and share information. The connection of billions of human minds must in my opinion lead to some kind of shared, common consciousness, although I cannot image what this really would encompass.

On the other hand, the number of machines with some kind of intelligence connected to the internet is growing at an amazing speed. The human brain contains 100 billion cells. Apparently this number is sufficient to give us consciousness. It is estimated that within the next five years there will be 15 billion devices that can be connected to the internet, of which 40 percent will actually be connected. And probably within 50 years the number of connected devices will exceed the number of brain cells. What might happen then? Will there be some kind of artificial consciousness? In any case it is likely that this complexity will result in emerging intelligent behaviour that is now difficult to imagine. The world will be surrounded by a noosphere of intelligence, both human and artificial.

What is the role of informatics in this development?

The field of informatics is quite young. Half a century ago, mathematicians, physicists, and electrical engineers started to build computers and to develop programming languages in order to let these machines perform required actions. Thus a new science was born, called computer science or informatics. In the early years, formal methods based on mathematics and straightforward logic formed the roots of this new science. Later on, many new braches sprouted from these roots, involved with computer graphics, multimedia, networks, communication, natural language processing, data mining, embedded software, virtual reality, human-computer interaction, etcetera.

And the scope of informatics is still expanding. The fast development of ICT continuously generates new research questions and problems that ask for a solution and the field of informatics is therefore facing tremendous challenges.

Three observations can be made about the future direction of informatics.

In the first place the complexity of ICT is growing to an extent that we can no longer rely on straightforward logic and formal methods to describe, understand and predict its behaviour. We have to cope with unexpected, emerging behaviour and will need new scientific approaches, based on experimentation and simulation. And I think that we need a new breed of informatics researchers with a more holistic systems view, besides the specialists in specific subdisciplines.

In the second place it becomes obvious that multidisciplinary co-operation is becoming essential. ICT is enabling breakthroughs in many other scientific fields, like for instance the life sciences. Researchers from the various disciplines have to work together to create these breakthroughs.

In addition, new applications of ICT generate new problems and undesired side effects which require different expertise. An example is computer crime. Informatics specialists have to co-operate with legal specialists to find solutions for such problems.

In the third place more co-operation is needed between academic research and developers in practice. ICT is the driving force of innovation in many domains and these innovations require a concerted effort of specialists in ICT as well as of specialists in the field of application. Innovations lead to new processes, business 
models and organisational models, and take place in open co-operative networks of academic research institutes, industry, business, government agencies, health care organisations etcetera. This so-called open innovation model is needed to really use ICT to its full potential and realise economic growth, higher quality of life and progress.

It took billions of years for the universe to develop complex molecules. It took a few billion years for life to develop from simple one-cell forms to mammals. It took millions of years from mammals to mankind, and something like a hundred thousand years for mankind to develop our modern industrialised society. Now we have witnessed the development of ICT in only half a century, and may experience exciting and fundamental changes within the next generation. Indeed, we live in interesting times!

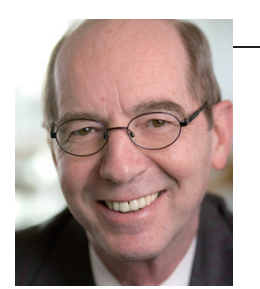

Gerard van OORTMERSSEN

Gerard van Oortmerssen is managing director of TNO Information and Communication Technology. TNO is the Netherlands Organization for Applied Scientific Research (www.tno.nl). Before he took this position in 2003, he has been for twelve years managing director of CWI, the national research institute for mathematics and computer science in Amsterdam. From 1998 till 2003 he was president of ERCIM, the European Research Consortium for Informatics and Mathematics. He is also board member of various startup companies in the field of ICT. 